\title{
Brain Imaging of Language Plasticity in Adopted Adults: Can a Second Language Replace the First?
}

\begin{abstract}
Do the neural circuits that subserve language acquisition lose plasticity as they become tuned to the maternal language? We tested adult subjects born in Korea and adopted by French families in childhood; they have become fluent in their second language and report no conscious recollection of their native language. In behavioral tests assessing their memory for Korean, we found that they do not perform better than a control group of native French subjects who have never been exposed to Korean. We also used event-related functional magnetic resonance imaging to monitor cortical activations while the Korean adoptees and native French listened to sentences spoken in Korean, French and other, unknown, foreign languages. The adopted subjects did not show any specific activations to Korean stimuli relative to unknown languages. The areas activated more by French stimuli than by foreign stimuli were similar in the Korean adoptees and in the French native subjects, but with relatively larger extents of activation in the latter group. We discuss these data in light of the critical period hypothesis for language acquisition.
\end{abstract}

\section{Introduction}

Young children seem to have a special gift for learning language. Research on early language acquisition has revealed that infants become attuned to the properties of their native language quickly (Werker and Tees, 1984; Halle et al., 1991; Kuhl et al., 1992). Moreover, several studies have established that the age of acquisition of a first or a second language is a major determinant of ultimate proficiency (Oyama, 1976; Newport, 1990; Mayberry and Eichen, 1991; Flege et al., 1995): a lag of even a few years in first exposure to a language can result in subtle but significant deficits in the command of its phonology, morphology or syntax (Johnson and Newport, 1989; Weber-Fox and Neville, 1996; Pallier et al., 1997). These facts have often been taken to support the existence of a critical, or sensitive, period for language learning in humans, similar to that observed in non-human species in other domains (Doupe and Kuhl, 1999).

One explanation attributes this to a progressive loss of plasticity of the neural circuits that subserve language learning. The change in plasticity may result from age-related maturational processes (Penfield, 1959; Lenneberg, 1967), or from the act of learning itself (Bever, 1981; Flege et al., 1999). However, this explanation, henceforth 'the crystallization hypothesis', is controversial: some late learners of a second language apparently become perfectly fluent speakers, and it remains an unsettled issue whether or not, provided adequate environment and training, the human brain has the potential to reach native-like command of a second language (Long, 1990; Birdsong, 1992; Werker and Tees, 1992; Strange, 1995; Harley and Wang, 1997; MacWhinney, 1997). The crystallization hypothesis predicts that the later a second language is learned, the more the cortical representations of the second and the first languages will differ. This prediction, however, has received only mixed support. In a functional magnetic resonance imaging (fMRI) study of sentence
C. Pallier ${ }^{1,2}$, S. Dehaene ${ }^{1}$, J.-B. Poline $^{1}$, D. LeBihan ${ }^{1}$, A.-M. Argenti ${ }^{2}$, E. Dupoux ${ }^{2}$ and J. Mehler $^{2}$

${ }^{1}$ INSERM U562, Service Hospitalier Fredrik Joliot, CEA/DSV/DRM, \& IFR49, 4 place du Général Leclerc, Orsay and ${ }^{2}$ Laboratoire de Sciences Cognitives et Psycholinguistique, EHESS-CNRS, 54 bd Raspail, Paris, France

production in bilinguals, consistent with the hypothesis, the patterns of activation elicited by internal production in L1 and L2 in Broca's area overlapped completely in early bilinguals, but differed in late bilinguals (Kim et al., 1997). However, in a subsequent study of single-word production, highly overlapping activations were found in early and late bilinguals (Chee et al., 1999b). In comprehension tasks, the ultimate level of proficiency in L2, more than the age of acquisition, seems to predict whether the cortical representations of L1 and L2 match (Dehaene et al., 1997; Perani et al., 1998; Chee et al., 1999a). In low-proficiency bilinguals, activations when listening to L2 vary greatly from one individual to another and often differ from those elicited by L1 (Dehaene et al., 1997). However, in a positron emission tomography (PET) study of a subset of late bilinguals who managed to become extremely fluent in L2, the cortical representations of $\mathrm{L} 1$ and $\mathrm{L} 2$ were indistinguishable and similar to those of native speakers (Perani et al., 1998). The latter findings seem at odds with the crystallization hypothesis. However, such a group study using PET may not be sensitive enough to detect subtle changes in the organization of language areas. Furthermore, it could be argued that the highly proficient participants, several of whom were professional simultaneous translators, were exceptional individuals.

Another prediction of the crystallization hypothesis is that exposure to the first language should leave long-lasting traces in the neural circuits subserving language processing. In the present study, we explore this issue by using fMRI to study the cerebral bases of speech comprehension in a special group of subjects: children adopted from foreign countries who have ceased to use their first language and speak only the language of their new parents. When tested as adults, they are apparently fluent in their second language and report having completely forgotten their first language (Maury, 1995). Has L2 completely replaced L1? According to the crystallization hypothesis, exposure to L1 during the first 3-8 years of life should have left traces. Exposing adopted subjects to sentences in their original language should elicit some remnant activity in language areas. If, on the other hand, the brain circuits for language remain plastic then learning a new language may have completely overridden the traces laid down by the first. To our knowledge, this is the first behavioral and neuroimaging study that uses evidence from adopted children to examine the plasticity of language acquisition when the child's main language suddenly ceases to be used.

\section{Materials and Methods}

\section{Subjects}

To find adopted subjects, we contacted several organisms in charge of foreign adoption in France, and sent mail to several hundreds of adopted subjects from various countries. From the answers we got back, the only sufficiently large group was formed of people of Korean origin. After 
excluding those who had had contacts with Korean since their arrival, and those who could not participate in the fMRI experiment for various reasons, we ended up with eight individuals for whom we had good evidence of sudden and definitive isolation from the initial maternal language (two females, six males ranging in age from 20 to 32, mean = 26.8; the ages of adoption were 3,3 , 5.5, 5.5, 5.5, 7, 7.5 and 8 years). All claimed to have completely forgotten their native language, as is generally the case for children adopted from foreign immigration (Maury, 1995) When interviewed about their skills in French, only one (who arrived at 5.5) reported significant problems in learning to speak French. The others reported having learned French rather quickly, and had no perceptible foreign accent in French.

Given their history of adoption, a concern is the extent to which those subjects' initial language acquisition was normal and, in particular, whether they had received sufficient inputs in the Korean language. Though we have little information on their individual history, the adopting organisms informed us that, in the orphanages they came from, they had interaction not only with other children but also with Korean-speaking adults. Older children went to Korean school. Thus, it is likely that they received a normal exposure to Korean. The control group comprised eight native monolingual French speakers (two females, six males ranging in age from 22 to 28 , mean $=23.5$ ), who had had no exposure to any Asian language. All subjects were right-handed according to the Edinburgh questionnaire.

All subjects participated in two behavioral tests out of the scanner, and one test inside the scanner.

\section{Behavioral Test 1: Language Identification}

The subjects listened to a total of 60 sentences, 12 in each of five languages unfamiliar to French subjects (Korean, Japanese, Polish, Swedish and Wolof). Sentences were read by three different female native speakers. After each sentence, the subject had to provide, on a scale from 1 to 7 , a degree of confidence that the sentence was in Korean or not: ' 7 ' meant that he/she was sure the sentence was in Korean; ' 1 ' meant that he/she was sure that the sentence was not in Korean; 4 indicated a complete lack of confidence.

\section{Behavioral Test 2: Word Recognition}

The experiment consisted of a series of 24 trials that started with the display of a word (written in French) followed by the auditory presentation of two Korean words. The task was to decide which of the two Korean words was the correct translation of the word displayed. Subjects could replay the Korean words as often as they wanted before responding

\section{fMRI Design and Acquisition}

Brain imaging was performed using event-related fMRI while the participants listened to a total of 128 sentences in four different languages: French, Korean, Japanese and Polish. Japanese and Polish were both unknown to our subjects, and thus served as a control for French and Korean sentences. In addition, Japanese was more similar to Korean than Polish, as attested by our pre-test. We were interested in assessing whether this difference in similarity would show up on the cortical activations.

Sentences were selected from recordings by three female native speakers of each language. The sentences were selected from a corpus of sentences from various languages, all translated from the same original French sentences. All recordings were made in the same sound-proof room (sampling rate $64 \mathrm{kHz}$; low-pass $20 \mathrm{kHz}$; $4 \times$ undersampling). The sentences, read with natural intonation, were 16-21 syllables long. They were selected so that the distributions of durations were similar across languages $($ average $=2.9 \mathrm{~s})$. The mean energies $(\mathrm{rms})$ of the stimuli were also equated.

To ensure that the subjects paid attention to the sentences, they were required to perform a fragment detection task. Following each sentence, after a $500 \mathrm{~ms}$ delay, a $400 \mathrm{~ms}$ fragment was played. The subject had to indicate, by pressing one of two response buttons, whether this fragment had appeared in the sentence or not. Before scanning, subjects performed a practice run of 12 trials on this task.

The auditory stimulations (playing one sentence followed by a probe) had a mean duration of $4 \mathrm{~s}$ and occurred every $19.6 \mathrm{~s}$, during time-windows where the read-out gradients were disabled, which greatly decreased the noise in the scanner. After each stimulation, six wholebrain scans were acquired ( $T_{2}{ }^{*}$-weighted echo-planar imaging, $3.75 \times$ $3.75 \times 5 \mathrm{~mm}, 22$ slices, $T_{\mathrm{R}}=2.4 \mathrm{~s}$, on a Bruker $3 \mathrm{~T}$ magnet $)$. The stimuli were administered in four 10 min blocks, each comprising 32 trials. Languages, speakers and side of response were evenly distributed within each block. The instruction (respond 'present' with the right or with the left hand) was switched between the second and third block. The sentences were presented in a different randomized order for each subject. A total of $128 \times 6=768$ functional images were acquired for each subject. High resolution $T_{1}$ anatomical scans (3-D gradient-echo inversionrecovery sequence, $T_{\mathrm{I}}=700 \mathrm{~ms}, T_{\mathrm{R}}=1600 \mathrm{~ms}, \mathrm{FOV}=192 \times 256 \mathrm{~mm}^{2}$, matrix $=256 \times 128 \times 256$, slice thickness $=1.2 \mathrm{~mm}$ ) were also acquired.

\section{fMRI Data Analyses}

fMRI data were processed using SPM99 software, starting with slice timing, spatial realignment (correction for movements), spatial normalization and smoothing with a $5 \mathrm{~mm}$ Gaussian kernel. For the statistical analyses, we generated a linear model by defining six categories of events: French, Korean, Japanese or Polish stimulations, and right or left motor responses (Friston et al., 1995). These categories were crossed with indicator variables for the four sessions, yielding a total of 24 onset vectors, which were convolved by an ideal hemodynamic impulse response and its derivative (included to model small temporal shifts). Both individual analyses and group analyses were performed; the group analyses consisted of $t$-tests using the individual contrast images smoothed at $8 \mathrm{~mm}$ (one image per subject). Unless otherwise indicated, the individual and group results were examined at the voxelwise threshold of $P<0.001$ uncorrected for multiple comparisons and the $P<0.05$ threshold on the extent of clusters.

\section{Results}

\section{Behavioral Tests}

In the sentence identification test, the native Korean subjects failed to recognize Korean sentences. Rather, their ratings were identical for Korean and Japanese sentences [4.09 and 4.04, respectively: $t(7)=0.30, P=0.70$ ] and were higher for those languages than for the other three languages presented: Polish, Swedish and Wolof $[t(7)=5.0, P=0.001]$. An analysis of variance indicated that this pattern of performance did not differ significantly from that observed in the group of native French participants [group $\times$ language interaction: $F(4,56)=1.60$, $P=0.17$; Fig. $1 a$ ]. In the second test, where subjects were asked to select the translation of a written French word from two spoken Korean words, again, the Korean and the control groups performed similarly $[56 \%$ and $52 \%$ success, respectively, $t(14)=0.98, P=0.30$; see Fig. $1 b$ ]. Finally, in the speech segment detection task performed during fMRI scanning, performance did not differ between the two groups [group $\times$ language interaction: $F(3,14)=1.5, P=0.22$ ]. Both Korean and control subjects showed better performance for the only language that they could understand (French) than for the other three languages $[t(7)=6.65, P<0.001$ and $t(7)=6.77, P<0.001$; Fig. $1 c]$. Inspection of individual data in the three tests showed that none of the Korean adoptees performed markedly differently from the control group.

\section{Brain Imaging}

Our first analysis aimed at identifying the network of areas that are more responsive to French than to other, unknown, languages. We choose the 'Polish' condition as a baseline that was neutral for the two groups, and determined the areas where French stimuli elicited stronger activations than Polish stimuli. The group analyses revealed a network of areas comparable to the one identified in previous studies of sentence listening (Mazoyer et al., 1993; Perani et al., 1996, 1998; Dehaene et al., 
a. Korean sentences identification

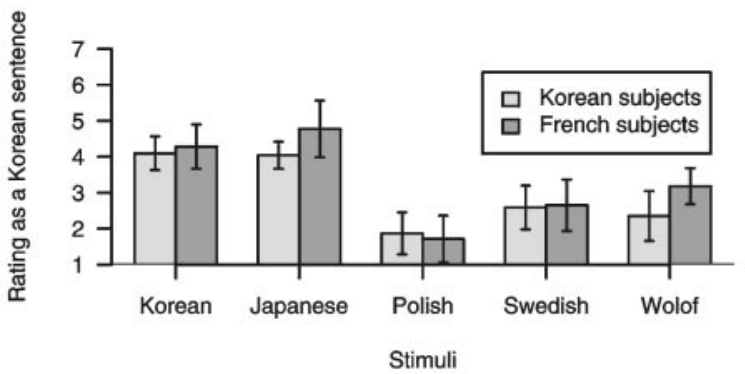

b. Word recognition

\section{c. Speech segment detection}
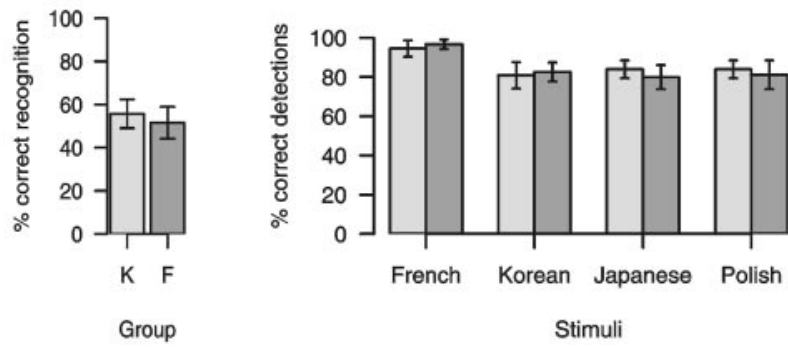

Figure 1. Performance of the native Korean and the native French subjects on three behavioral tests. In the 'Korean sentence identification' test (a), subjects listened to sentences in five different languages and rated their confidence that the sentences were in Korean $(7=100 \%$ sure it is Korean; $4=$ totally unsure, $1=$ sure it is not Korean). In the 'Word recognition test' (b), the subjects saw a French word and had to select the correct translation amongst two spoken Korean words. In the 'segment detection task' (c), subjects had to decide whether a short sound excerpt did or did not belong to a sentence they had just heard. In all tests, the Koreans' performance did not differ from that of the control group (error bars display $95 \% \mathrm{CI}$ ).

1997; Schlosser et al., 1998). This network comprised the left STS and neighboring portions of the superior and middle temporal gyrus, the left inferior frontal gyrus (triangularis sector), and, to a lesser extent, the contralateral temporal areas of the right hemisphere. Similar networks were activated in both groups of subjects (see Table 1, Figs $2 A$ and 3 ): direct comparison of the two groups yielded no significant difference (even when the threshold was decreased to $P<0.01$ voxelwise uncorrected for multiple comparisons and the cluster size threshold set to $P<0.05$ ). Similar results were obtained when the activations evoked by Japanese sentences, rather than Polish sentences, were used as the baseline (Fig. 2B).

Inspection of the individual analyses of the French-Polish comparison (Fig. 4) revealed that while the participants in both groups activated similar areas, those from the Korean group had smaller extents of activations than the control group. Counts of voxels above the $P<0.001$ threshold were submitted to an analysis of variance with the factors 'hemisphere' and 'native language' (see Fig. 5): both factors were significant [Group: $F(1,14)=7.3, P=0.02$; Hemisphere: $F(1,14)=24.0, P<0.001]$ but did not interact $[F(1,14)=1.6, P=0.23]$. A similar analysis for counts of voxels above $P<0.05$ corrected, yielded a similar outcome. Importantly, no such group difference was found when we compared the extents of motor cortex activations associated with left-hand and right-hand key presses [Group: $F(1,14)=0.53, P=0.48]$. This suggests that the lesser extent of activation in Korean subjects during language listening was not due to changes in anatomical organization, head shape or imaging parameters, but was possibly related to their peculiar history of language acquisition.
Table 1

Areas where activations were greater for French than for Polish stimuli, for the French and Korean subjects

\begin{tabular}{|c|c|c|c|c|}
\hline & $x$ & y & $z$ & $t$-value \\
\hline \multicolumn{5}{|l|}{ French subjects } \\
\hline Left STS anterior & -56 & -8 & -16 & 11.94 \\
\hline Left STS anterior & -60 & 4 & -24 & 5.28 \\
\hline Left STS middle & -56 & -32 & -4 & 10.68 \\
\hline Left STS middle & -52 & -24 & -8 & 6.64 \\
\hline Left STS posterior & -52 & -48 & 12 & 9.32 \\
\hline Left STS posterior & -44 & -60 & 16 & 6.51 \\
\hline Left STS posterior & -40 & -48 & 24 & 5.83 \\
\hline Left MTG posterior & -60 & -44 & -4 & 8.24 \\
\hline Left MTG middle & -68 & -24 & -16 & 7.01 \\
\hline Left MTG middle & -64 & -20 & -12 & 6.96 \\
\hline Left temp. pole & -52 & 12 & -20 & 5.88 \\
\hline Left IFG (pars triang.) & -56 & 24 & 0 & 9.16 \\
\hline Left IFG (pars triang.) & -44 & 20 & 16 & 5.87 \\
\hline Right STS middle & 48 & -20 & -12 & 7.55 \\
\hline Right STS anterior & 52 & -4 & -16 & 6.75 \\
\hline \multicolumn{5}{|l|}{ Korean subjects } \\
\hline Left STS anterior & -60 & -12 & -16 & 8.69 \\
\hline Left STS anterior & -52 & 4 & -24 & 11.78 \\
\hline Left STS middle & -52 & -36 & -8 & 6.78 \\
\hline Left STS middle & -56 & -20 & -12 & 7.40 \\
\hline Left STS posterior & -60 & -56 & 8 & 4.68 \\
\hline Left MTG posterior & -64 & -40 & -4 & 4.64 \\
\hline Left MTG posterior & -64 & -48 & -8 & 3.70 \\
\hline Left ITG posterior & -68 & -52 & -16 & 5.00 \\
\hline Left temp. pole & -48 & 12 & -24 & 10.9 \\
\hline Left IFG (pars triang.) & -56 & 24 & 0 & 6.24 \\
\hline Left IFG (pars triang.) & -56 & 20 & 12 & 3.97 \\
\hline Right STS middle & 52 & -28 & -12 & 4.46 \\
\hline Right STS anterior & 52 & 4 & -20 & 6.33 \\
\hline Right temp. pole & 48 & 20 & -32 & 3.82 \\
\hline
\end{tabular}

We report the coordinates in millimeters and $t$-values of the local maxima of activations identified in the group analyses. Thresholds: $t_{7}>4.8, P<0.001 ; t_{7}>3.0, P<0.01$. MTG, middle temporal gyrus; IFG, inferior frontal gyrus; ITG, inferior temporal gyrus.

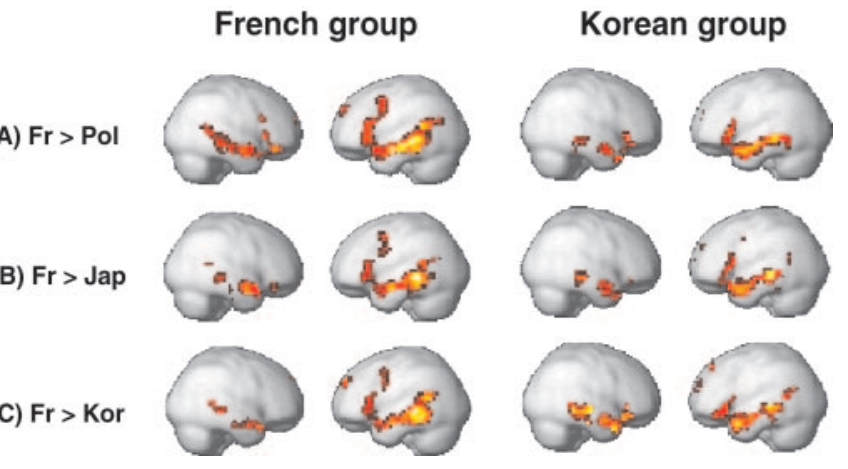

Figure 2. Brain renderings displaying the results in the group analyses of the three contrasts comparing French stimuli versus Polish stimuli $(A)$, Japanese stimuli $(B)$ and Korean stimuli $(C)$.

Next we examined the presence of activations specific to Korean sentences, again relative to Polish sentences. In the Korean group, no significant difference was found (at $P<0.01$ or at $P<0.001$ voxelwise uncorrected for multiple comparisons, and $P<0.05$ corrected for cluster extent). Furthermore, in the individual analyses, none of the native Korean subjects showed any Korean-specific activations $(P<0.001$ uncorrected $)$. In the group analyses of native French speakers, however, two regions were significantly more activated by Korean than by Polish stimuli: one in the left cerebellum [maximum at $-12,-56,-16$; 


\section{Korean Ss}
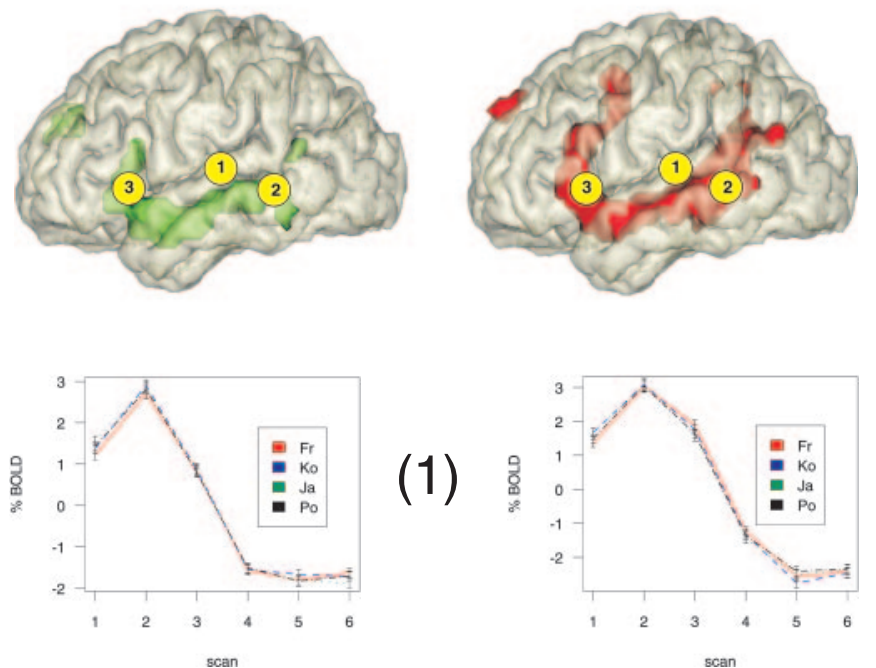

(1)

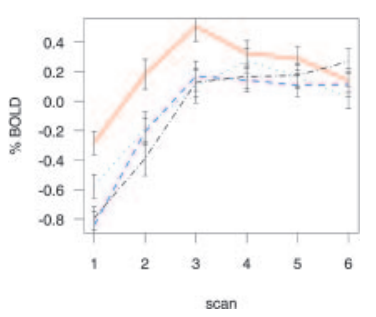

(2)

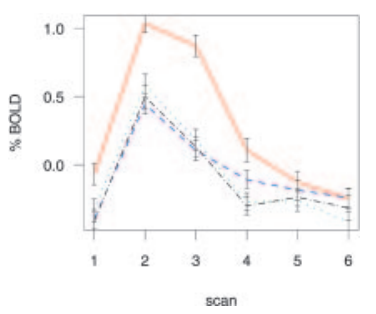

(3)
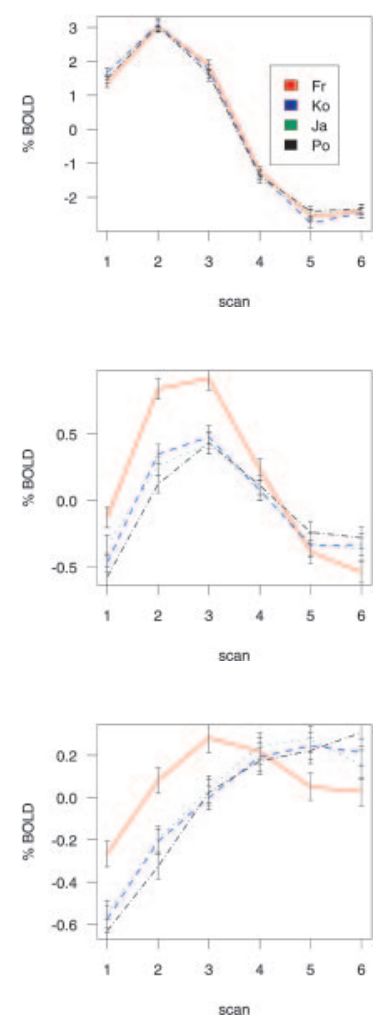

Figure 3. Time-course of activations in three different regions of the left hemisphere $(1=$ Heschel gyrus, 2 = posterior STS, $3=$ inferior frontal gyrus). The percentage changes in signal are shown as a function of the scan position following sentence presentation (time between scans $=2.4 \mathrm{~s}$ ). The four different types of line correspond to each of the languages in which the stimuli were spoken (French, Korean, Japanese and Polish). For each group, the maximum peak in the region of interest was selected.

$T(7)=7.08]$ and one in the right middle superior temporal sulcus (STS) [at $44,-12,-12 ; T(7)=18.24]$. When the two groups were compared, this right STS region came out significantly when the threshold was lowered at $P<0.01$ [at 48, -20, -12; T(14) $=5.35$ ] However, a cluster of activation located about the same region $[\max$. at $48,-4,-8 ; T(14)=4.74]$ was also detected in the Korean group in the 'Korean-Polish' analysis at the $P<0.01$ voxel level (but this did not approach the $P<0.05$ extent threshold). This may be related to differences between Korean and Polish which were perceptible to the participants (see Fig. 1a). Yet, similar analyses contrasting Korean and Japanese sentences, and Japanese and Polish sentences did not reveal any significant difference between the languages or the two groups.

Finally, we compared the activations to French versus Korean sentences directly (Fig. 2C). In the French group, this revealed the same left-hemispheric network identified in the 'French >
Polish' contrast. If Korean subjects had any remnant trace of having learned Korean as the first language and French as the second, then the French versus Korean difference should be attenuated or even reversed in this group. This was not the case. The French versus Korean contrast was highly significant along the left STS and in left inferior frontal areas in the Korean group, just as in the French group. No brain area showed any group difference in the 'French > Korean' nor in the 'Korean > French' contrasts.

\section{Discussion}

We studied language perception and comprehension using behavioral methods and fMRI in a group of Korean adults adopted in their youth by French families, as well as in a control group of native French subjects. Three main results were observed. First, behaviorally, the adopted subjects could not distinguish sentences in their native language from sentences coming from various languages. Nor could they identify Korean words in a forced-choice task, or detect fragments from Korean sentences better than native French controls. Second, the fMRI data revealed no differences in brain activation when the adopted subjects listened to Korean relative to an unknown language such as Polish or Japanese. Third, the cortical regions that responded more to the known language, French, than to other foreign languages were similar in the adopted subjects and in the native French control group. However, the observed activations in this comparison had a broader extent in the native French subjects than in the adopted subjects.

These data do not support a strong version of the crystallization hypothesis. Indeed, this hypothesis led us to expect activations specific to Korean in the adopted Koreans, and a differential pattern of activated areas between the French and Korean groups, while listening to French. Actually, the activation patterns in the Koreans were remarkably similar to those of the native French group, at least in terms of the regions that were activated. Slight differences were detected in the extent and amount of activation; these are further discussed below. In both groups, there was a large activation of left-lateralized temporal and inferior frontal regions when listening to French sentences, but essentially no detectable activation of this network when listening to Korean sentences relative to two other, unknown, languages. This provides evidence in favor of the reversibility of plastic changes associated with language acquisition in the first few years of life. The subjects in our study had been adopted between 3 and 8 years of age. If the brain circuits that subserve language acquisition had started to crystallize during this time period, we would have expected to see larger differences between the two groups. Our data rather suggest that when a second language is learned early on, this acquisition does not necessarily involve different brain systems than those involved in learning the native language. On the contrary, the second language may become represented in the very areas that normally represent the first language. This conclusion fits with a previous PET study of spoken language comprehension in which late but highly fluent learners of a second language were found to have patterns of activation indistinguishable from those of native speakers (Perani et al., 1998). Compared with this previous study, the present study has the advantage that the adopted subjects were not selected because they were exceptionally gifted for foreign languages. Our study suggests that any child, if placed in the unusual situation of having to learn a new language between 3 and 8 years of life, can succeed to a high degree, and that they do so using the same brain areas as are recruited for first-language acquisition. 


\section{French Ss}
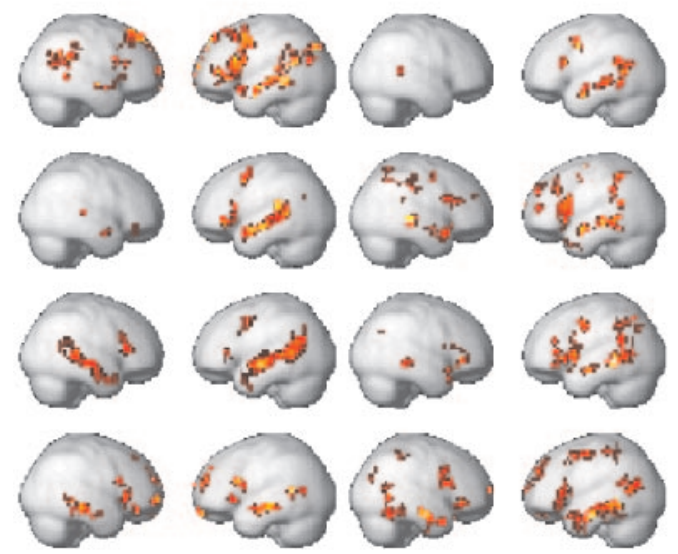

Korean Ss
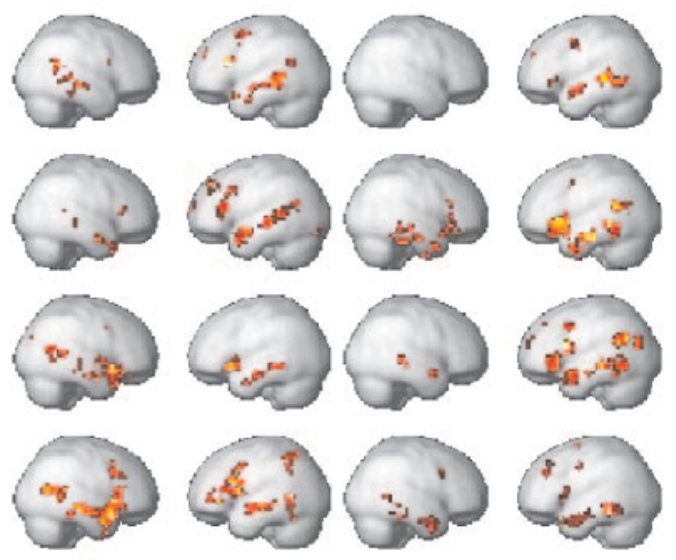

Figure 4. Brain renderings showing, for each of the 16 subjects, the regions where activation due to French sentences was significantly stronger $(P<0.001)$ than that due to Polish sentences.

\section{French subjects}

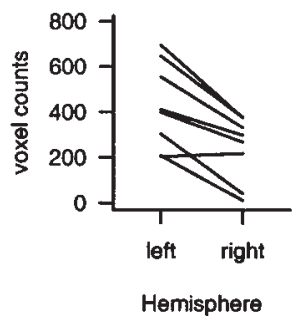

Korean subjects

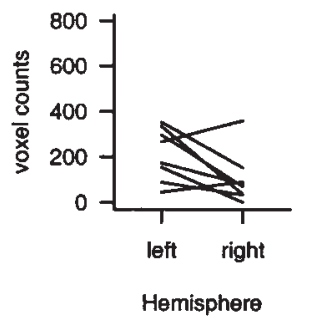

Figure 5. Numbers of supra-threshold $(P<0.001)$ voxels in individual statistical parametric maps for the 'French-Polish contrast', split by hemispheres (each line corresponds to one subject).

The behavioral observations that we collected also support the adopted subjects' claim that they have forgotten their native language. On a Korean sentence identification test, they did not perform any better than naive French subjects. This was true also in the lexical test that involved selecting the appropriate meaning for common words, and in the speech segment detection test. One limitation of these tests (except possibly for the third) is that they were not designed to test for subtle, implicit, remnants of Korean in the adopted subjects. Early experience with Korean may have left implicit unconscious traces at the level of the microcircuitry of the language processing areas that our behavioral and fMRI methods did not detect. If they exist at all, however, such traces must be small and perhaps take the form of 'dormant' synaptic changes that cannot be revealed with classical fMRI subtraction methods. Such traces might be revealed by using a learning paradigm. For example, more extensive retraining experiments (which could not be performed in the half-day visit on the present study) might reveal faster learning of Korean in subjects who had early exposure to Korean relative to control subjects, as has been observed in both animals and humans (Tees and Werker, 1984 Knudsen, 1998; Au et al., 2002). We are in the process of devising such a training test, to assess whether the adopted Koreans can perceive phonetic contrasts that exist in Korean but not in French better than native French listeners.

Our fMRI experiment used natural sentences in order to reveal potential traces of Korean in the adopted subjects at any of

different linguistic levels, from phonology to the lexicon, prosody, syntax and semantics. To isolate such traces, we subtracted the activation to Korean stimuli from the activation to stimuli in two foreign languages, Japanese and Polish. This procedure has previously been shown to reveal a large network of activation when processing a known relative to a foreign language (Mazoyer et al., 1993; Dehaene et al., 1997; Schlosser et al., 1998). However, one difficulty with this subtraction logic is that some areas, particularly those involved in speech decoding and phonological processes, may be subtracted away because they may be equally well activated by known and unknown languages. Indeed, the absence of differential activations in the superior temporal gyrus, which has been associated with speech decoding (Hickok and Poeppel, 2000), in both the adopted subjects and the control group, might be interpreted as supporting the idea that foreign stimuli engage phonological processes as much as do native stimuli. Thus, the subtractive method may have been suboptimal to identify traces of the first language in the early stages of speech processing.

Although the bulk of our results suggests a high degree of similarity between adopted subjects and native French subjects, we found two differences between the groups worth discussing. First, when listening to French relative to foreign stimuli, although the same anatomical regions were activated, the extent of activation was larger in the native French participants relative to the Korean adopted subjects. This result speaks to the main question that our experiment was designed to address: can the second language replace the first? The results discussed above imply that it can, but this latter finding suggests that such replacement may not be complete. The acquisition of expertise is known to be accompanied by local increases in brain activation (Gauthier et al., 1999; Poldrack and Gabrieli, 2001) and an enlargement of cortical maps (Elbert et al., 1995) concomitant with a narrowing of cortical tuning (Rainer and Miller, 2000). The native French subjects' greater experience with French may have resulted in a widening of the cortical maps for language processing, and/or an increase in their responsivity, in comparison to the Korean subjects. Though we have informally observed that the adopted Koreans have a very good command of French, subsequent research will be needed to assess whether they might differ from native speakers in subtle ways.

Alternatively, the relative difference in the extent of activation 
between groups, could be due to larger activation in the adopted subjects by the foreign stimuli. Contrary to the control group, which was composed of naive students from the Paris area, the adopted subjects had agreed to participate in the study because of their adopted status, and were coming to Orsay from other parts of France specifically for this study. Given their natural curiosity for their special status, we cannot exclude the possibility that they deployed greater attention to the foreign language stimuli than the control subjects. It is also possible that their unusual history of language acquisition has caused a greater responsivity of left-hemispheric language areas to any form of linguistic input. At present, we cannot discriminate between the above explanations.

A second difference between the groups was observed in the Korean-Polish subtraction, which revealed an activation in the right STS that was stronger in the native French group than in the adopted subjects. This unpredicted result may be due to a relative inhibition in the Koreans relative to the French group, when listening to Korean. This interpretation raises the issue of the possible role of inhibition in the forgetting of the first language. However, the fact that the two groups did not differ in the Korean-Japanese and French-Korean substractions, mitigates this interpretation.

We close by considering the possible implications of this research for theories of critical periods in language acquisition. The original hypothesis put forward by Lenneberg (Lenneberg, 1967) stipulates that the critical period for language ends around puberty. Given that the adopted subjects arrived in France when they were less than 8 years of age, our results are not incompatible with Lenneberg's hypothesis. However, another version of the critical period hypothesis holds that there is a gradual loss of plasticity starting much before puberty. This view builds on the fact that language perception becomes attuned to the linguistic environment very early on in life. Developmental research has shown that even children in their first year already show the influence of their surrounding linguistic environment in both their behavior (Werker and Tees, 1983; Halle et al., 1991; Kuhl et al., 1992) and their brain activations (Dehaene-Lambertz and Dehaene, 1994; Dehaene-Lambertz, 1997; Näätänen et al., 1997; Cheour et al., 1998). This is consistent with studies of second-language processing, which indicate that in immigrants, even a lag of a few years in the onset of second-language acquisition is associated with perceptible deficits compared to native subjects. Such deficits are most evident in speech production, where a foreign accent starts to be perceptible when a second language is acquired after the age of 4 years (Oyama, 1976; Flege et al., 1995). Similar studies on speech comprehension also reveal deficits in syntax in Chinese immigrants who arrived in the USA in their third year (Weber-Fox and Neville, 1996).

It is often not realized that this sort of empirical finding can be submitted to two radically different theoretical interpretations. It is useful to distinguish between interference and 'crystallization' accounts of the critical period hypothesis. Both accounts agree that language acquisition starts very early on, probably guided by genetically driven mechanisms partly dedicated to processing speech inputs. However, they differ in their interpretation of subjects' difficulties in acquiring a second language. According to the crystallization account, a window of brain plasticity is open at birth and progressively closes as the brain networks for language become stabilized, under the possible influence of maturational and/or experiential factors. In this view, plasticity is temporally limited and progressively lost. According to the interference account, on the other hand, the presence of processes and representations attuned to the first language acts as a filter that distorts the way a second language can be acquired. In this view, the loss of plasticity in language areas, if it exists at all, plays only a minor role compared to the interference imposed on the maintenance of a first language by the processing of the second.

The study of adopted children provides a unique opportunity to address this theoretical distinction. Contrary to immigrants, who usually stay in contact with their home family and local language community, adopted children do not need to maintain any representation of the first language, from which they are suddenly deprived. In this situation, our data tentatively suggest that the native language is, in large part, lost and replaced by the language of the new environment. Even by 7 or 8 years of age, plasticity in language areas is still sufficiently high to promote an essentially complete recovery of normal language. This conclusion fits with lesion studies that found good, if incomplete, recovery from large left hemisphere lesions, or even from left hemispherectomy when performed before the age of 9 (Vargha-Khadem et al., 1997). Our results complement these studies by showing that this form of plasticity is not limited to exceptional situations of brain insult or intractable epilepsy, but that it also occurs in the normal brain. This view is not incompatible with the notion that puberty is associated with a biologically determined reduction in language learning ability (Lenneberg, 1967). Indeed, previous brain-imaging studies have revealed major differences in the cortical representation of the first and second languages in most late learners (Perani et al., 1996; Dehaene et al., 1997; Kim et al., 1997). We might obtain similar results if we could study a population of subjects who had been delocalized to a new country and severed from their home language late in life, after puberty. Nor are our data incompatible with developmental evidence for early brain changes associated with language acquisition. Our results merely indicate that those early changes are not immediately stabilized, but remain plastic and reversible for several years.

\section{Notes}

We thank the participants and the organizations in charge of foreign adoption who helped us. We thank F. Hennel for help in data acquisition. This work was supported by an ACI grant from the Ministère de la Recherche awarded to C. Pallier and a joint European project of the European Commission, DG XII.

Address correspondence to C. Pallier, INSERM U562, SHFJ CEA, 4 place du Général Leclerc, Orsay, France, F91401. Email: pallier@lscp.ehess.fr.

\section{References}

Au TK, Knightly LM, Jun SA, Oh JS (2002) Overhearing a language during childhood. Psychol Sci 13:238-243.

Bever T (1981) Normal acquisition processes explain the critical period for language learning. In: Individual differences in language learning aptitude (Diller K, ed.), pp. 176-198. Rowley, MA: Newbury House.

Birdsong D (1992) Ultimate attainment in second language acquisition. Language 68:708-755.

Chee M, Caplan D, Soon C, Sriram N, Tan E, Thiel T, Weekes B (1999a) Processing of visually presented sentences in Mandarin and English studied with fMRI. Neuron 23:127-137.

Chee M, Tan E, Thiel T (1999b) Mandarin and English single word processing studied with functional magnetic resonance imaging. J Neurosci 19:3050-3056.

Cheour M, Ceponiene R, Lehtokoski A, Luuk A, Allik J, Alho K, Näätänen R (1998) Development of language-specific phoneme representations in the infant brain. Nature Neurosci 1:351-353.

Dehaene S, Dupoux E, Mehler J, Cohen L, Paulesu E, Perani D, van de Moortele PF, Léhericy S, LeBihan D (1997) Anatomical variability in the cortical representation of first and second languages. Neuroreport 8:3809-3815.

Dehaene-Lambertz G (1997) Electrophysiological correlates of categorical perception in adults. Neuroreport 8:919-924. 
Dehaene-Lambertz G, Dehaene S (1994) Speed and cerebral correlates of syllable discrimination in infants. Nature 370:292-295.

Doupe A, Kuhl P (1999) Birdsong and human speech: common themes and mechanisms. Annu Rev Neurosci 22:567-631.

Elbert T, Pantev C, Wienbruch C, Rockstroh B, Taub E (1995) Increased cortical representation of the fingers of the left hand in string players. Science 270:305-307.

Flege JE, Munro MJ, MacKay IR A (1995) Factors affecting strength of perceived foreign accent in a second language. J Acoust Soc Am 97:3125-3134.

Flege JE, Yeni-Komshian GH, Liu S (1999) Age constraints on secondlanguage acquisition. J Mem Lang 41:78-104.

Friston KJ, Holmes AP, Worsley KJ, Poline JB, Frith CD, Frackowiak RSJ (1995) Statistical parametric maps in functional imaging: a general linear approach. Hum Brain Mapp 2:189-210.

Gauthier I, Tarr MJ, Anderson AW, Skudlarski P, Gore JC (1999) Activation of the middle fusiform 'face area' increases with expertise in recognizing novel objects. Nature Neurosci 2:568-573.

Halle P, de Boysson-Bardies B, Vihman M (1991) Beginnings of prosodic organization: intonation and duration patterns of disyllables produced by Japanese and French infants. Lang Speech 34:299-318.

Harley B, Wang W (1997) The critical period hypothesis: where are we now? In: Tutorials in bilingualism: psycholinguistic perspectives (de Groot AMB, Kroll JF, eds), pp. 19-51. Mahwah, NJ: Lawrence Erlbaum Associates.

Hickok G, Poeppel D (2000) Towards a functional neuroanatomy of speech perception. Trends Cogn Sci 4:131-138.

Johnson JS, Newport EL (1989) Critical period effects in second language learning: the influence of maturational state on the acquisition of English as a second language. Cognit Psychol 21:60-99.

Kim KHS, Relkin NR, Lee KM, Hirsch J (1997) Distinct cortical areas associated with native and second languages. Nature 388:171-174

Knudsen E (1998) Capacity for plasticity in the adult owl auditory system expanded by juvenile experience. Science 279:1531-1533.

Kuhl P, Williams K, Lacerda F, Stevens K, Lindblom B (1992) Linguistic experience alters phonetic perception in infants by 6 months of age. Science 255:606-608.

Lenneberg EH (1967) Biological foundations of language. New York: Wiley.

Long M (1990) Maturational constraints on language development. Stud Sec Lang Acquisit 12:251-285.

MacWhinney B (1997) Second language acquisition and the competition model. In: Tutorials in bilingualism: psycholinguistic perspectives (de Groot AMB, Kroll JF, eds), pp. 113-142. Mahwah, NJ: Lawrence Erlbaum Associates.

Maury F (1995) Les mécanismes intrapsychiques de l'adoption internationale et interraciale. l'adoption des enfants coréens en france. PhD thesis, Université de Paris VIII, Paris.

Mayberry R, Eichen EB (1991) The long-lasting advantage of learning sign language in childhood: another look at the critical period for language acquisition. J Mem Lang 30:486-512.

Mazoyer BM, Dehaene S, Tzourio N et al. (1993) The cortical representation of speech. J Cogn Neurosci 5:467-479.

Näätänen R, Lehtokoski A, Lennes M et al. (1997) Language-specific phoneme representations revealed by electric and magnetic brain responses. Nature 385:432-434.

Newport EL (1990) Maturational constraints on language learning. Cogn Sci 14:11-28.

Oyama S (1976) A sensitive period for the acquisition of a nonnative phonological system. J Psycholinguist Res 5:261-283.

Pallier C, Bosch L, Sebastian-Gallés N (1997) A limit on behavioral plasticity in speech perception. Cognition 64:B9-B17.

Penfield W (1959) Speech and brain mechanisms. Princeton, NJ: Princeton University Press.

Perani D, Dehaene S, Grassi F, Cohen L, Cappa S, Dupoux E, Fazio F, Mehler J (1996) Brain processing of native and foreign languages. Neuroreport 7:2439-2444.

Perani D, Paulesu E, Sebastian N, Dupoux E, Dehaene S, Bettinardi V, Cappa SF, Fazio F, Mehler J (1998) The bilingual brain. Proficiency and age of acquisition of the second language. Brain 121:1841-1852.

Poldrack RA, Gabrieli JDE (2001) Characterizing the neural mechanisms of skill learning and repetition priming: evidence from mirror reading. Brain 124:67-82.

Rainer G, Miller EK (2000) Effects of visual experience on the representation of objects in the prefrontal cortex. Neuron 27:179-189.

Schlosser MJ, Aoyagi N, Fulbright RK, Gore JC, McCarthy G (1998) Functional MRI studies of auditory comprehension. Hum Brain Mapp 6:1-13.

Strange W, ed. (1995) Speech perception and linguistic experience. Baltimore, MD: York Press.

Tees RC, Werker JF (1984) Perceptual flexibility: maintenance or recovery of the ability to discriminate non-native speech sounds. Can J Psychol 38:579-590.

Vargha-Khadem F, Carr L, Isaacs E, Brett E, Adams C, Mishkin M (1997) Onset of speech after left hemispherectomy in a nine-year-old boy. Brain 120:159-182.

Weber-Fox CM, Neville HJ (1996) Maturational constraints on functional specializations for language processing: Erp and behavioral evidence in bilingual speakers. J Cogn Neurosci 8:231-256.

Werker JF, Tees RC (1983) Developmental changes across childhood in the perception of non-native speech sounds. Can J Psychol 37:278-286.

Werker JF, Tees RC (1984) Cross-language speech perception: evidence for perceptual reorganization during the first year of life. Infant Behav Dev 7:49-63.

Werker JF, Tees RC (1992) The organization and reorganization of human speech perception. Ann Rev Neurosci 15:377-402. 